\title{
TÉCNICA Y ACTUALIDAD DE LA URODINÁMICA. PROBLEMAS Y RETOS
}

\author{
J. ROMERO MAROTO, L. PRIETO CHAPARRO
}

Servicio de Urología. Unidad de Urodinámica. Hospital Universitario de San Juan. San Juan (Alicante).

Actas Urol Esp. 27 (2): 75-91, 2003

\section{RESUMEN}

"TÉCNICA Y ACTUALIDAD DE LA URODINÁMICA. PROBLEMAS Y RETOS"

$\mathrm{El}$ presente artículo pone de manifiesto el avance experimentado por la urodinámica fundamentalmente en la última década, así como los problemas a los que tendrá que hacer frente en el futuro: investigación en la etiopatogenia de diferentes enfermedades, buscar procedimientos diagnósticos más sensibles y específicos que los actuales, establecer indicaciones más exhaustivas para las exploraciones urodinámicas y con ello, conseguir el perfeccionamiento de determinados procedimientos quirúrgicos.

Se detalla la técnica e interpretación de las diferentes exploraciones urodinámicas a un nivel muy actual, así como las novedades existentes, las dificultades de aplicación y controversias. La neuromodulación y la urodinámica ambulatoria merecen detalle en este artículo. Finalmente se muestran las controversias y retos de futuro, como son la investigación urodinámica que proporcione de forma precisa el diagnóstico de la obstrucción del tracto urinario inferior en la mujer, establecer la indicación del estudio urodinámico en la mujer con incontinencia urinaria de esfuerzo, perfilar las indicaciones de la neuromodulación y de la urodinámica ambulatoria, aplicabilidad de sistemas de inteligencia artificial, mejorar los materiales del esfinter artificial, desarrollo de tejidos para realizar ampliaciones vesicales y la prevención real de la mielodisplasia.

PALABRAS CLAVE: Urodinámica. Aplicaciones. Indicaciones. Técnica.

\section{ABSTRACT}

"URODYNAMICS TECHNIQUE AND CURRENT SITUATION. ISSUES AND CHALLENGES"

Development of urodynamics particularly during the past decade are highlighted, as well as a number of issues to be faced in the near future: research into the etiopathogenesis of different conditions, finding more sensitive and specific diagnostic procedures to overcome the current ones, establishing more comprehensive indications for urodynamics examinations and, as a result of all the above achieving greater improvement of certain surgical procedures.

Both the technique and interpretation of the different urodynamic examinations, as well as the more recent innovations, implementation issues and controversies are detailed to a highly up-to-date level. Neuromodulation and ambulatory urodynamics deserve thoughtful consideration in this paper. Finally, attention is given to the controversies and future challenges such as urodynamic research providing accurate diagnosis of lower urinary tract obstruction in women, establishing the indication of urodynamic studies in women with urinary exertional incontinence, outlining the indications of neuromodulation and ambulatory urodynamics, applicability of artificial intelligence systems, improvement of artificial sphincter materials, tissue growth for bladder enlargement ad actual prevention of myelodysplasia.

KEY WORDS: Urodynamics. Applications. Indications. Technique. 
$\mathrm{L}^{2}$ a urodinámica, en un sentido amplio, puede dentenderse como la subespecialidad de la Urología que estudia la dinámica del aparato urinario y las distintas enfermedades que alteran dicho comportamiento. Tendríamos así, por una parte, el estudio de dichas enfermedades y por otra, las distintas exploraciones que permiten estudiar la dinámica y sus alteraciones, que conducen, a su vez, al diagnóstico de dichas enfermedades.

El interés por la urodinámica ha crecido de forma espectacular en las últimas tres décadas, apoyado sobre todo en el gran desarrollo tecnológico experimentado (Figs. 1, 2 y 3), que ha aportado instrumentos de valoración objetiva. Esto ha supuesto el planteamiento y el estudio de muchos de los problemas existentes concernientes con la urodinámica, el esclarecimiento de algunos y la certeza de que las exploraciones urodinámicas son imprescindibles para el diagnóstico y consiguiente tratamiento correcto de una determinada serie de enfermedades.

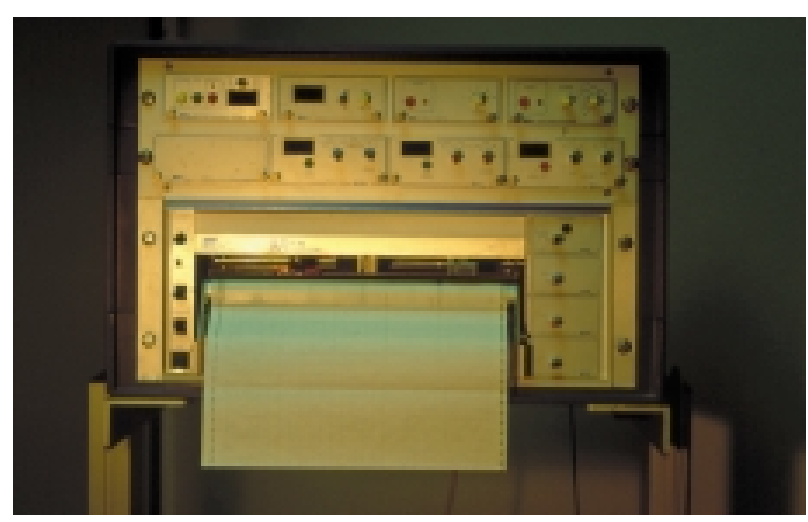

FIGURA 1. Equipo de urodinámica antiguo de papel térmico.

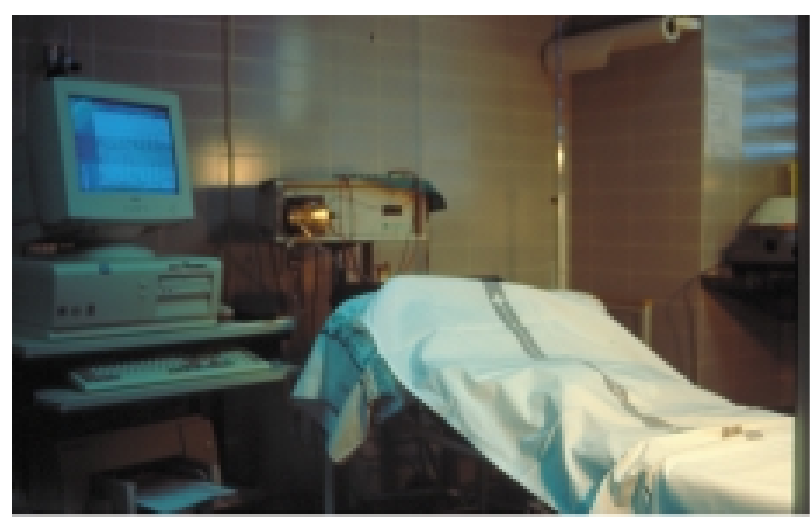

FIGURA 2. Moderno equipo de urodinámica actual.

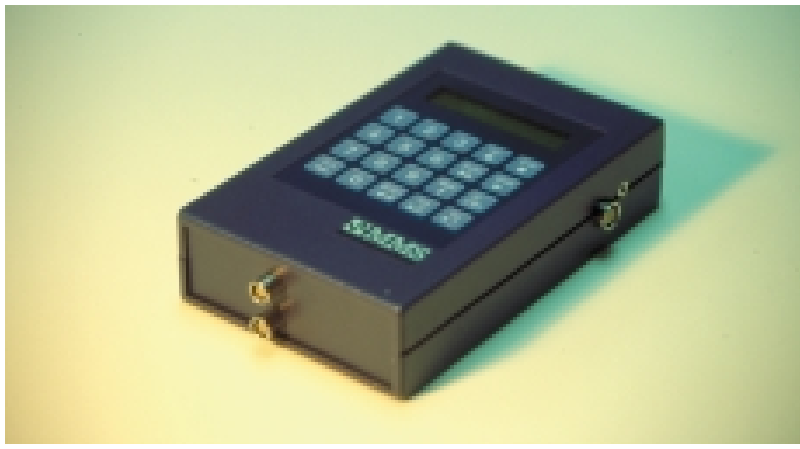

FIGURA 3. Equipo de urodinámica ambulatoria de reducidas dimensiones.

La ponencia sobre vejiga neurógena de Don Luis Cifuentes en 1966, el curso de disfunción miccional del Dr. Martínez Piñeiro en 1973 y la ponencia sobre Urodinámica de los Dres. Azagra, Resel y Solé en 1974, posiblemente marquen el inicio de la Urodinámica moderna en España ${ }^{1}$. La creación del Grupo Español de Urodinámica por el Dr. Ponce de León, en 1979, agrupa a una serie de jóvenes urólogos interesados en el tema. Desde entonces, las unidades de urodinámica han experimentado un crecimiento espectacular en nuestro país y hoy el 90\% de los Servicios de Urología disponen de equipo de urodinámica.

En nuestro Servicio, para un Área de Salud de 200.000 habitantes, realizamos 250 estudios urodinámicos al año, haciéndolo el $66 \%$ en mujeres y el $44 \%$ en hombres. El 47,6\% de estudios se realiza en mujeres adultas, cuyo motivo de consulta es el estudio de incontinencia urinaria de esfuerzo, de urgencia o mixta, en el 18,1\% en relación a disfunción neurógena vésicouretral en adultos, el 16,8\% para evaluar el diagnóstico de obstrucción en el varón con síntomas del tracto urinario inferior, el 10,8\% para evaluar en niños con vejiga neurógena congénita, en el 5,1\% incluye, micción no coordinada, enuresis, reeducación vesical, y finalmente el 1,2\% el diagnóstico es no reproducción de síntomas.

Estos porcentajes de reparto no cambian significativamente con lo que se estudiaba en otros centros de referencia 15 años atrás ${ }^{2}$, lo que si ha cambiado substancialmente es el número de indicaciones de estudio urodinámico por 100.000 habitantes de área de Salud.

A pesar del avance experimentado por la Urodinámica, existen aún una serie de problemas a los que tendrá que hacer frente en el futuro. 
Básicamente son:

- El esclarecimiento de los mecanismos etiopatogénicos de distintas enfermedades, que faciliten un tratamiento adecuado.

- El hallazgo de procedimientos diagnósticos más sensibles que los actuales, que permitan diagnósticos no posibles hoy día.

- El establecimiento de indicaciones correctas de determinadas exploraciones y la determinación del valor exacto de las mismas.

- El perfeccionamiento de ciertos procedimientos quirúrgicos.

Las técnicas urodinámicas usadas están hoy, a pesar de sus limitaciones, realmente consolidadas, existiendo cierta controversia sobre la aplicación y la indicación de algunas. Las diversas exploraciones no excluyen una historia y una exploración física correctas, que son las que orientan y determinan el tipo de prueba que se realizará. El paciente debe conocer siempre en que consiste la exploración indicada y qué se espera conseguir de ella.

\section{FLUJ OMETRÍA}

La flujometría forma parte de las exploraciones iniciales de todo paciente con sintomatología del tracto urinario inferior ${ }^{3}$. Es la exploración más sencilla en urodinámica, la cual a pesar de sus limitaciones, representa la forma más orientativa de valorar la micción. Consiste en la determinación de varios parámetros al medir la evacuación urinaria por unidad de tiempo, lo que nos permite obtener el flujo miccional, volumen de orina y tiempo de micción fundamentalmente. Es rápida, económica y fácilmente reproducible. No es una exploración invasiva y proporciona una información muy aproximada de la fase miccional sin molestias para el paciente.

La micción realizada en el gabinete de urodinámica, para ser valorable, debe reproducir la micción habitual del paciente. El paciente ha de tener un deseo miccional normal y orinar en privado. El entorno ha de proporcionar al paciente la máxima tranquilidad, confidencialidad y evitar en lo posible cualquier interrupción que pueda suponer una interferencia psicológica. Asimismo, deben orinar en su posición habitual, ya que muchos varones, por diversas cuestiones pueden hacerlo sentado de forma habitual. La valoración del residuo post-miccional, bien por cateterismo o por ecografía completa el estudio. La realización de tres flujometrías ofrece mayor fiabilidad de la prueba $^{4}$.

\section{Flujometría ambulatoria}

La flujometría ambulatoria puede ofrecer mayor exactitud que la que se realiza en el gabinete de urodinámica. Se ha propuesto la flujometría ambulatoria con el fin de obtener una idea más exacta del flujo miccional real del enfermo, dado que existen flujómetros portables de fácil manejo. Se consigue también con los modernos monitores que incorporan flujómetro ambulatorio o con incluso determinaciones de conductancia uretral, capaces de medir y almacenar diversas micciones en 24 horas para su posterior análisis gracias a una batería y un microprocesador incorporados ${ }^{5}$. Está por establecerse sus indicaciones concretas, aunque es un método útil para aquellas personas incapaces de orinar en la unidad de urodinámica debido a influencias psicológicas o de otro orden.

\section{Flujometría con manguito}

La realización de la flujometría con un manguito ${ }^{6}$ pretende obtener un registro que aproxime el valor de la presión isovolumétrica del detrusor, para de forma indirecta conocer datos de la capacidad contráctil del detrusor, prescindiendo del sondaje vesical y minimizando la invasividad. Otros autores han tratado de medir esta presión mediante la oclusión repentina con un catéter balón o mediante interrupción voluntaria brusca o Stop-test ${ }^{7}$, aunque sólo determina parámetros uretrales y no la capacidad contráctil del detrusor. Está demostrada la fiabilidad del re-test. No se ha extendido su uso en las Unidades de Urodinámica.

\section{Flujometría con EMG}

La flujometría libre puede asociarse con $\mathrm{EMG}^{8}$, para estudiar las disfunciones detruso-esfinterianas. Si el enfermo emplea prensa abdominal para la evacuación urinaria se puede artefactar el resultado, incrementándolo ${ }^{9-12}$. Debido a ello, algunos autores han incorporado la utilización de un tocodinamómetro para registrar la actividad muscular abdominal durante la micción ${ }^{13}$. Se debe instruir a los pacientes para tratar de no utilizar prensa durante la micción ${ }^{14}$. Es una exploración muy útil 
y poco agresiva en las diferentes disfunciones miccionales en la infancia. Nos da una visión clara de la situación miccional. Lo usamos también como bio-feed-back en rehabilitación funcional vesical con unos resultados bastante aceptables.

\section{Flujometría con radionúclidos}

La flujometría con radionúclidos es una técnica simple, no invasiva y perfectamente comparable a la flujometría convencional. Además de aportar todos los parámetros de vaciado convencionales, permite incluso la medición del volumen residual sin necesidad de otros exámenes complementarios ${ }^{15-19}$. No obstante, la necesidad de incorporar estos sofisticados y caros equipos a un gabinete de urodinámica no justifica los beneficios que se obtienen.

\section{Flujometría con dióxido de carbono}

Por último, otra modificación técnica es la realización de la flujometría con dióxido de carbono ${ }^{20}$. Este método determina el flujo en función de la compresión neumática del dióxido de carbono. Los valores obtenidos con este método son similares a los comunicados en la literatura por otras técnicas. También en este caso el flujo es volumen dependiente y cuando el volumen vaciado es mayor de $200 \mathrm{ml}$, los valores de flujo máximo son equiparables a la flujometría convencional.

\section{CISTOMANOMETRÍA}

La cistomanometría consiste en determinar mediante un procedimiento reglado, los cambios de presión que experimenta la vejiga urinaria, con el incremento progresivo de volumen intravesical, siendo representado todo ello en un registro gráfico.

La cistomanometría es una exploración invasiva relativamente libre de complicaciones, sin embargo no es inocua, existiendo notables excepciones ${ }^{21}$. Su realización requiere tener adecuada información clínica sobre el enfermo en el que se va a practicar y ha de tener relevancia en el manejo de la patología sospechada, razón por la que se indica.

Pretende reproducir los síntomas urinarios que refiere el enfermo en la fase de llenado vesical, obteniendo unos parámetros cualitativos y cuantitativos. Sin un criterio académico bien asentado, su indicación en población asintomática es discutible.
Los catéteres urodinámicos para la realización de estudios pueden ser de dos tipos, catéteres para transductores de agua, que conducen la presión de columna de agua y de microtip, es decir, de membrana en el terminal del catéter mucho más sensibles y costosos. Para la práctica clínica los de agua son los más utilizados, usándose los de microtip en experimentación y en urodinámica ambulatoria.

La cistomanometría trata de reproducir el síntoma en fase de llenado, por ello el enfermo ha de tener si es posible la posición de decúbito, bipedestación, deambulación y sedestación. La fijación de catéteres, prolongadores adecuados que no artefacten la señal y cadencia de movimientos adecuados permiten generalmente ésto.

\section{Registro de resultados e interpretación}

El objetivo fundamental de la cistomanometría es conseguir la reproducción del síntoma y su evaluación en términos analíticos mensurables. Toda contracción vesical mientras se realiza el llenado en una cistomanometría es anormal. La International Continence Society estableció en 15 cm de $\mathrm{H}_{2} \mathrm{O}$ la presión mínima para realizar el diagnóstico de una contracción inestable o hiperrefléxica del detrusor ${ }^{22}$. Blaivas ${ }^{23}$, entre otros, ha reconocido el significado de una contracción de menor magnitud, que puede presentarse en casos de insuficiencia de la capacidad contráctil del detrusor. Por otra parte, la definición de esta hiperactividad vesical está condicionada por el test que intenta demostrar las contracciones involuntarias en la cistomanometría y su reproductibilidad (diferentes maniobras de provocación, posición del enfermo, no reproducción de síntomas, etc...).

Las contracciones involuntarias del detrusor o hiperactividad vesical son reconocidas por la aparición en el llenado de contracciones fásicas (incremento de presión) cuando el paciente trata de inhibirlas. Por otra parte, la definición matemática básica de la distensibilidad se basa en el cambio que experimenta la presión vesical conforme cambia su volumen, entendido como una contracción tónica durante el llenado ${ }^{24}$. Además, la capacidad total de la vejiga es un reflejo indirecto de la distensibilidad aunque en ella no se tienen en cuenta los cambios de la tensión de la vejiga a nivel de su pared. Podemos ver ejemplos en 
las Figuras 4, 5, 6 y 7 de cistomanometría normal, vejiga de baja acomodación, hiperreflexia vesical con disinergia e inestabilidad vesical respectivamente.

El valor absoluto de la presión del detrusor en la contracción no es el único determinante de alteración funcional, pues también es importante el tiempo durante el cual actúan estas presiones contra la resistencia uretral.

\section{Punto de presión de fuga}

A finales de los años $70^{25}$, dos grupos de diferentes centros, observaron que los niños con mielodisplasia, cuya incontinencia se producía con presiones detrusorianas superiores a $40 \mathrm{~cm}$ de $\mathrm{H}_{2} \mathrm{O}$, acababan presentando sistemáticamente enfermedad del tracto urinario superior si no se les trataba. Se comprobó que en estos casos, existía una vejiga muy poco distensible. La aparición de enfermedad del tracto superior era mucho menos frecuente en aquellos enfermos cuya presión vesical de fuga era inferior a $40 \mathrm{~cm} \mathrm{de} \mathrm{H}_{2} \mathrm{O}$.

Este concepto de presión de pérdida de líquido se ha visto ampliado a otras situaciones y en base a otros principios fisiopatológicos: si la pérdida de líquido es ocasionada por una determinada presión, si es posible independizar el origen de éstas, este criterio podría ser aplicable a otras entidades, como la incontinencia urinaria femenina de esfuerzo. Así pues, en los últimos años se ha introducido en urodinámica el concepto de presión de fuga en la cistomanometría, que alude fundamentalmente a la presión intravesical que es capaz de producir pérdida urinaria. Por ello, se definen conceptualmente tres tipos de presión de fuga:

1. La Presión Mínima de Escape en el Valsalva es un parámetro ${ }^{26}$ que cuantifica específicamente la presión abdominal o presión vesical (de Valsalva) total en ausencia de contracción del detrusor, necesaria para causar incontinencia urinaria de esfuerzo.

2. La presión abdominal de fuga, parámetro derivado del anterior también utilizado en la evaluación de la incontinencia urinaria de esfuerzo, al no conseguirse en algunos casos presiones elevadas con el Valsalva, con semejante utilidad según algunos autores ${ }^{27}$.

3. La presión vesical de fuga, que se refiere a la presión de fuga por contracción del detrusor que mide la resistencia del mecanismo esfinteriano o del conducto de salida. A mayor presión de fuga mayor resistencia. Este parámetro es de gran utilidad en la evaluación y pronóstico de pacientes con vejiga neurógena, puesto que si es elevada, supone riesgo para el tracto urinario superior.

\section{Determinación de la presión de fuga}

Antes de su determinación, se necesita comprobar rigurosamente el residuo post-miccional para asegurarnos que comenzamos el estudio con la vejiga completamente vacía. La finalidad de esta medición es obtener la presión con la que se fuga la orina y ésta puede ser variable con diferentes volúmenes vesicales, por lo tanto, se hace imprescindible comenzar el llenado con la vejiga absolutamente vacía.

Se debe llenar la vejiga hasta capacidades que producen el escape o hasta que se obtiene el volumen máximo mediante el cateterismo intermitente. El llenado vesical se ha de realizar a una velocidad de $50 \mathrm{ml} / \mathrm{min}$ en adultos y a $20 \mathrm{ml} / \mathrm{min}$ en niños, como en la cistomanometría convencional.

La acomodación vesical es un parámetro muy importante para la determinación. Si la acomodación vesical está disminuida, la presión de pérdida de líquido que obtendremos tiene valor de presión de detrusor, de tal manera, que tendremos que independizar el componente de presión abdominal y obtendremos el punto de presión de fuga o presión vesical de fuga. De otra parte, si la acomodación es normal y no se demuestra actividad involuntaria del detrusor, lo que obtendremos con Valsalva, tos o prensa abdominal será presión abdominal de fuga.

Se ha de observar constantemente el meato uretral para objetivar la fuga, el instante y la presión con la que se produce. Esto ha de ser anotado en los diferentes episodios de escape urinario.

En procesos neurógenos, el estudio finaliza cuando la presión del detrusor es superior claramente a $40 \mathrm{~cm} \mathrm{de} \mathrm{H}_{2} \mathrm{O}$, se produce escape, se obtiene el volumen máximo del cateterismo intermitente o se produce contracción del detrusor ${ }^{25}$.

La determinación de la presión abdominal de fuga requiere una sistemática diferente, ya que como el objetivo es evaluar la presión abdominal de fuga, ésta puede acontecer a diferentes capacidades vesicales y a diferentes presiones abdominales, 


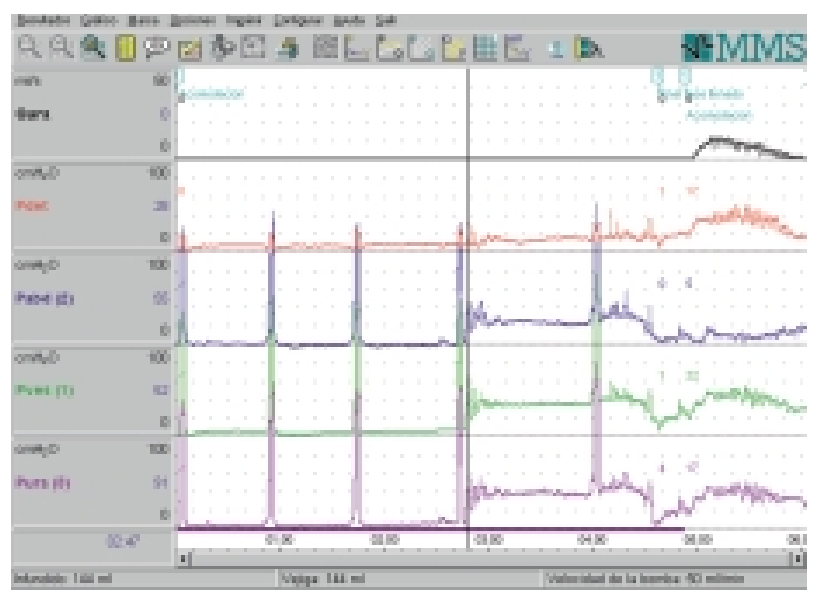

FIGURA 4. Cistomanometria normal.

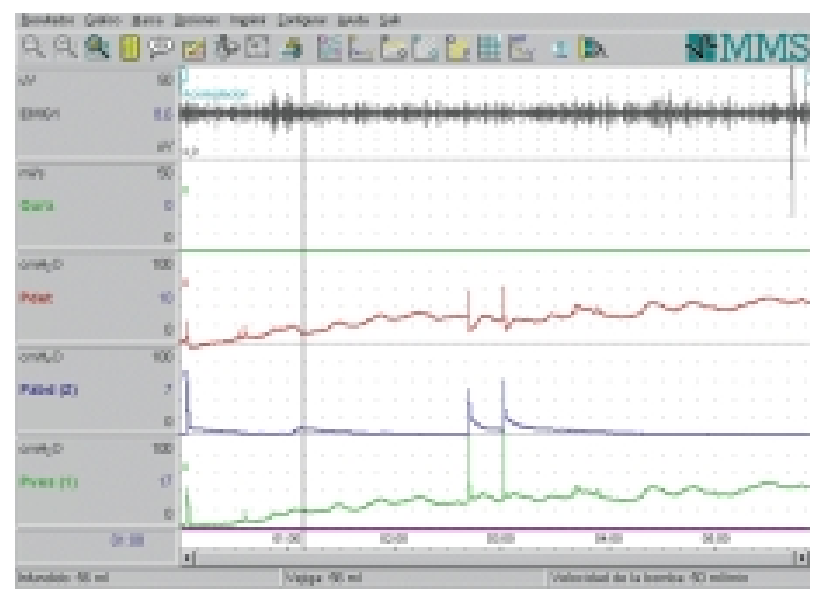

FIGURA 5. Cistomanometria en vejiga de baja acomodación.

por ello se solicitará conseguir a capacidades estandarizadas (cada $50 \mathrm{ml}$ de infusión) presiones abdominales menores a $30 \mathrm{~cm} \mathrm{de} \mathrm{H}_{2} \mathrm{O}$, menores a $60 \mathrm{~cm} \mathrm{de} \mathrm{H}_{2} \mathrm{O}$ y superiores a $90 \mathrm{~cm} \mathrm{de} \mathrm{H}_{2} \mathrm{O}$, lo que se consigue fácilmente indicando soplar, toser con intensidad moderada o toser fuerte.

En la Figura 8 se muestra un ejemplo de determinación de presión abdominal de fuga.

La determinación de la presión abdominal de fuga está afectada por determinadas condiciones:

\section{1) Volumen vesical}

El volumen vesical se correlaciona inversamente con la presión abdominal de fuga. A mayor volumen vesical en el momento de la determinación, menor presión abdominal de fuga. Se ha estudiado la presión de fuga a diferentes volúmenes, estableciéndose como volumen vesical recomendado aquel comprendido entre 250 y $300 \mathrm{ml}$.

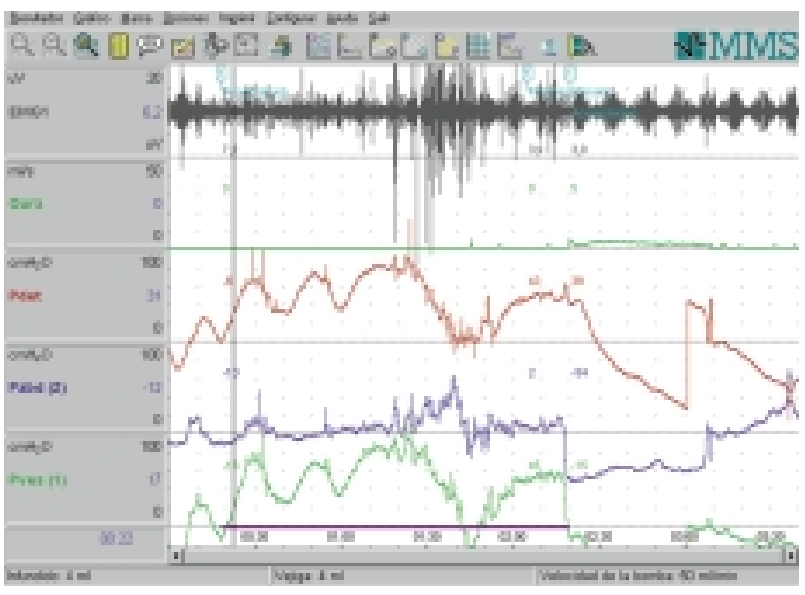

FIGURA 6. Cistomanometria en hiperreflexia vesical con disinergia.

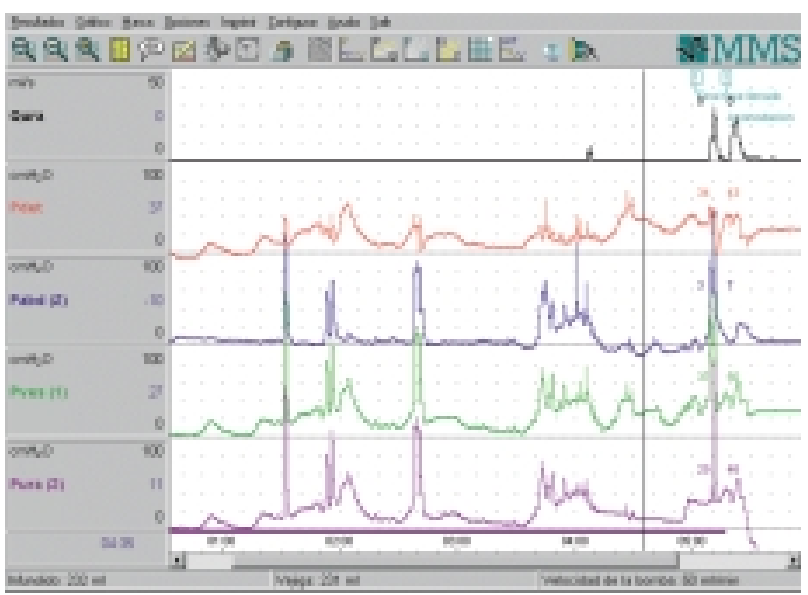

FIGURA 7. Cistomanometria en inestabilidad vesical.

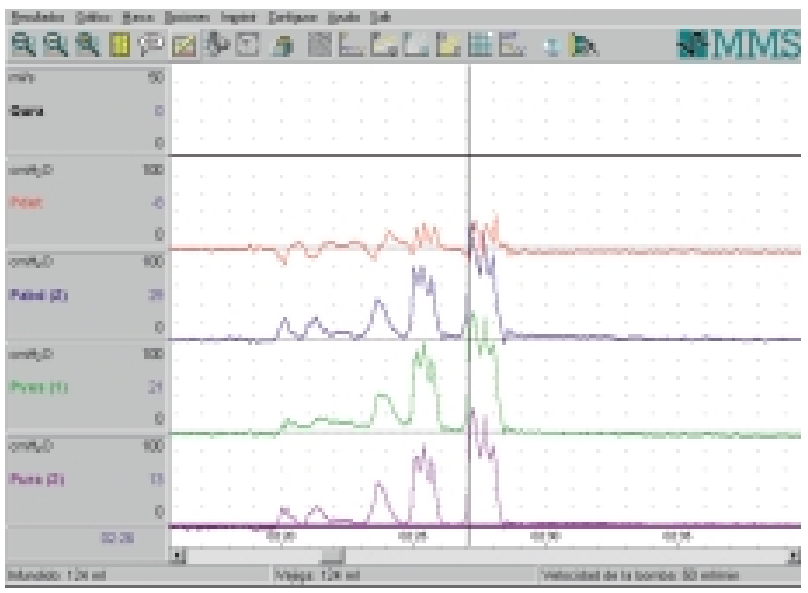

FIGURA 8. Determinación de presión abdominal de fuga.

La presencia de escape de orina a volúmenes bajos de perfusión, indica una incontinencia urinaria más severa que si éstos ocurren a volúmenes de perfusión más elevados. 
2) Calibre del catéter uretral

El incremento del calibre del catéter uretral causa un incremento en el valor de la presión abdominal de fuga ${ }^{28}$.

\section{3) Método de provocación}

Se utilizan dos tipos de maniobra de provocación para determinar la presión abdominal de fuga. Una es la maniobra de Valsalva, quizás la más utilizada y la otra sería la tos. Se han comparado las cifras de presión abdominal de fuga obtenidas a diferentes volúmenes de perfusión, obtenidas por cada una de estas maniobras. La obtenida mediante la tos es significativamente superior al obtenido por medio de la maniobra de Valsalva $^{9}$, tanto a $150 \mathrm{ml}$, como a $300 \mathrm{ml}$ o a 400 $\mathrm{ml}$. La maniobra de Valsalva sería el mejor método de provocación para obtener la mínima presión abdominal de fuga. Durante la tos, habría una respuesta refleja del periné y el esfínter uretral externo, que probablemente afectaría el valor de dicha determinación elevándola. En nuestra experiencia la realización de ambas no se excluyen puesto que en Valsalva resulta a veces difícil obtener elevadas presiones, no así con la tos. También preconizamos su realización en diferentes posiciones en la cistomanometría.

\section{4) Localización del catéter}

Se ha demostrado que el registro de presión abdominal ofrece los mismos resultados, tanto si se utiliza el incremento de presión vesical generado por el abdomen, como si se registra en el recto o en vagina ${ }^{29}$.

\section{5) Edad}

La edad y el estado menopáusico de la paciente no influyen en los resultados de la determinación de la presión abdominal de fuga ${ }^{28}$.

\section{6) Relación de la presión de fuga con el grado de} incontinencia

Se ha demostrado una buena correlación entre el grado clínico de incontinencia urinaria y la presión abdominal de fuga ${ }^{30-32}$. Así, las pacientes con incontinencia urinaria grado 1 presentaron una presión abdominal de fuga superior a $90 \mathrm{~cm} \mathrm{H}_{2} \mathrm{O}$ en todos los casos. El 75\% de las pacientes con incontinencia por déficit esfinteriano intrínseco tenían una presión abdominal de fuga inferior a $90 \mathrm{~cm} \mathrm{H}_{2} \mathrm{O}$ y en el $50 \%$ de ellas era inferior a $50 \mathrm{~cm} \mathrm{H}_{2} \mathrm{O}^{32}$.
7) Relación presión de fuga y máxima presión uretral de cierre del perfil

Antes de la aparición de la presión abdominal de fuga, se había establecido que un valor de la máxima presión uretral de cierre (MPUC) del perfil uretral inferior o igual a $20 \mathrm{~cm} \mathrm{H}_{2} \mathrm{O}$ suponía una incompetencia esfinteriana. Se ha demostrado una correlación lineal simple entre los valores del MPUC del perfil uretral y la presión abdominal de fuga ${ }^{33,34}$.

8) Relación de la presión de fuga con la hipermovilidad cérvico-uretral

Las pacientes con un aumento de movilidad del complejo cérvico-uretral tienen una presión abdominal de fuga significativamente más elevada que aquellas sin hipermovilidad ${ }^{32}$.

\section{9) Reproductibilidad}

Si se utiliza el mismo método en la determinación de la presión abdominal de fuga, el resultado es altamente reproducible ${ }^{25-27}$. La determinación de la presión abdominal de fuga participa de la estandarización propia de la cistomanometría. Bajo esta perspectiva y aunque no afectan por sí mismas a esta medida, hay que incluir la velocidad de perfusión, las características y temperatura del líquido de perfusión y la posición de la paciente.

10) Limites de presión abdominal de fuga

Para el perfil uretral una MPUC menor de 20 cm $\mathrm{H}_{2} \mathrm{O}$ establece la incompetencia esfinteriana, consiguiendo una aceptable especificidad. El problema que tiene la determinación de la presión abdominal de fuga es establecer un punto de corte, con el fin de aumentar su especificidad sin pérdida de sensibilidad. Acotando como en nuestra serie el déficit esfinteriano intrínseco a pérdidas menores con $30 \mathrm{~cm} \mathrm{H}_{2} \mathrm{O}$ de presión se es mucho más estricto con este diagnóstico, siendo considerados el $100 \%$ de los casos deficiencia intrínseca.

\section{Perfil uretral}

Otro de los métodos utilizados para estudiar el grado de competencia uretral son los Perfiles de Presión Uretral, exploración controvertida con defensores y detractores, pero que en opinión de algunos autores ${ }^{35,36}$ tiene un importante valor para determinar el grado de competencia esfinteriana en la mujer con incontinencia urinaria de estrés y especialmente cuando esta exploración se realiza con microtransductores ${ }^{37,38}$. 
La presión abdominal necesaria para originar incontinencia urinaria de esfuerzo es independiente de la presión uretral. En el pasado, la función uretral respecto a la continencia estaba basado en medidas de la presión uretral de cierre o cambios en la presión uretral máxima y de la longitud uretral funcional. Por otra parte estas presiones no reflejan la función uretral intrínseca aislada, su medición es artefactual y no reproducible ante los incrementos de presión ${ }^{32}$. La utilización de un sensor de presión en un tejido colapsado con las propiedades viscoelásticas de la uretra, hace que con microtransductores obtengamos una magnitud resultado de fuerzas de fricción, y no de presión ejercida por área de microtransductor, en rangos de pocos milímetros cuadrados. Por otra parte, la infusión de fluido, no necesariamente distiende la uretra como para proporcionar medidas de presión sin incluir fricción. Además, el registro puede presentar más variaciones artefactuales, por rotación del catéter, movimiento del mismo por su peso, etc.. La perfusión del catéter pudiera en ocasiones proporcionar una medición válida si existe alta distensibilidad uretral y perfecto cierre. Ante un cierre uretral imperfecto, la resistencia al flujo en esas irregularidades invalida los resultados ${ }^{37}$.

Una revisión crítica de los diferentes test y técnicas empleadas en el estudio de la afectación esfinteriana intrínseca nos hace ver que, el perfil uretral estático es una técnica de baja reproductibilidad, artefactual y con un valor predictivo más que cuestionable. Sin embargo, hay estudios en los que se ha encontrado un discreto grado de correlación, aunque no estadísticamente significativa, entre los valores de la MPUC y los de la presión abdominal de fuga. Han apreciado un mayor grado de correspondencia entre la intensidad de la incontinencia urinaria y los valores de la MPUC, que con los valores de la presión abdominal de fuga. No pueden establecer, a priori, basándonos exclusivamente en el valor de la presión abdominal de fuga el grado de competencia esfinteriana, al no haber diferencias significativas de la MPUC para distintos valores de la anterior determinación.

La realidad es que la división entre incontinencia esfinteriana por hipermovilidad e incontinencia por deficiencia intrínseca es cierta pero muy difícil de demostrar clínicamente. Sólo en aquellos casos en los que ha existido radioterapia previa, múltiples intervenciones quirúrgicas anteriores en un intento de corregir la incontinencia o existe disfunción neurógena es clara la posible afectación intrínseca. En el resto de los casos, ni histopatológicamente ni mediante E.M.G. se ha comprobado que una presión de fuga inferior a 60 cm $\mathrm{H}_{2} \mathrm{O}$ se corresponde con una afectación uretral. Existen todavía puntos sin aclarar en la etiopatogenia de la incontinencia de esfuerzo en la mujer.

\section{Aplicación en la incontinencia urinaria de la mujer adulta}

La determinación de la presión de fuga en el estudio de la incontinencia urinaria de esfuerzo en la mujer es un parámetro urodinámico incorporado para su estudio en la última década. Aún con problemas en su definición, reproductibilidad, utilidad y fiabilidad, no deja de ser común su determinación, sustentando en muchos centros, la orientación y tipificación terapéutica, fin último de la urodinámica. Paralelamente, sigue actualmente viva, la continua controversia entre urodinamistas que ven mayor utilidad frente al perfil uretral dinámico. Estas controversias todavía alimentan las discusiones y comentarios de reuniones científicas.

En la última década se incorpora a la terminología, conceptos como vejiga hiperactiva, incluyendo trastornos de la fase de llenado, excluyendo de este complejo sintomático las infecciones del tracto urinario y la cistitis intersticial. Lo más importante que se debe discernir de esta terminología, a nuestro entender desafortunada y dirigida, es cribar la hiperactividad sintomática, de la hiperactividad urodinámica no sintomática y de la hiperactividad sin demostración urodinámica.

Dado que la tendencia moderna de la medicina, es poner más énfasis en la prevención primaria de algunas enfermedades o síntomas, ya que es más barato, y posiblemente mejor que otros tratamientos, la reeducación y la rehabilitación del suelo pelviano en las unidades de urodinámica ha experimentado una profusión en esta última década, consiguiendo muy buenos resultados en algunos centros. La crítica también surge a estas tendencias, en lo que se refiere al control riguroso de 
resultados a largo plazo. Sirva de ejemplo, una de las series más representativas al respecto ${ }^{39}$ que evalúa resultados a 10 años vista sobre 87 enfermas, sometidas a ejercicios de Kegel del suelo pelviano; sólo en ese tiempo son evaluables 52 casos y de ellas se refiere mejoría-curación (no sólo curación, pues el criterio mejoría incluye la persistencia de incontinencia ocasional o leve) de la incontinencia urinaria, existiendo un porcentaje de intervenciones quirúrgicas no despreciable en dicha serie. De otra parte, cierto es, que en el caso en el que se haya conseguido curación mediante esta metodología, no se precisó intervención quirúrgica. La estricta selección de casos para ello también precisará mejor las indicaciones de ejercicios del suelo pélvico en el tiempo.

\section{Aspectos técnicos en casos especiales}

La práctica de la urodinámica se verá modificada de acuerdo con la información y características de los enfermos en los que se ha de realizar. Así pues, distinguimos los siguientes supuestos:

1. Población pediátrica. La cooperación y colaboración para realizar el estudio en estos pacientes resulta difícil, siendo en algunos casos imposible ganarse la confianza del niño o niña. La presencia de los padres durante el estudio puede suponer una ayuda tanto para realizar el estudio, como para justificar la imposibilidad de realizarlo. Los registros de presión obtenidos al chillar o llorar el niño si se dan alteraciones urodinámicas pueden ser difícilmente interpretables en algunos casos. La sedación con benzodiazepinas en los niños pueden alterar el estudio en lo que se refiere a la relajación del músculo estriado del esfínter externo.

2. Pacientes con disfunción neurógena vesicouretral. El espasmo de adductores en pacientes con espina bífida, puede dificultar el cateterismo. El catéter rectal puede ser innecesario cuando el tono anal es absolutamente laxo en estos enfermos. En enfermos sin micción espontánea o con residuo post-miccional importante es conveniente una vez terminada la cistomanometría evacuar el residuo post-miccional y una cobertura antibiótica es recomendada en estos casos. La disrreflexia autonómica con hipertensión severa resulta de la manipulación del tracto urinario inferior en algunos pacientes con vejiga neurógena, y consecuen- temente se requiere monitorizar la presión arterial en pacientes de riesgo. La premedicación con alfabloqueantes puede ser una ayuda en muchos de estos enfermos.

3. Esfinter artificial. Es importante conocer el funcionamiento del mismo, donde está el reservorio y el dispositivo de desactivación para el sondaje. En algunos casos se requiere estudio radiológico previo para comprobar estos extremos. El esfínter urinario artificial también ha quedado posicionado en esta década, no estando justificado a nuestro criterio su empleo, en el tratamiento de la incontinencia urinaria femenina, dada la depuración que han experimentado las técnicas de sling-cabestrillo-TVT, sobre todo acompañadas necesariamente de la corrección quirúrgica oportuna y simultánea de otros defectos de soporte ginecológicos asociados, si existen. Persisten otras indicaciones en incontinencia post-prostatectomía radical (siendo cada vez menos frecuente la incontinencia posterior) o en disfunciones neurógenas vesicouretrales.

4. Anormalidades anatómicas. Aquellas que afecten a la uretra pueden requerir si es imprescindible el estudio su realización vía suprapúbica. En las resecciones abdómino-perineales de recto la sonda rectal se emplaza por la colostomía, cuidando cerrar debidamente con adhesivos el espacio entre sonda rectal y estoma, obteniéndose registros similares que con la sonda emplazada en el ano. En las mujeres puede emplazarse la sonda en el fondo vaginal con igual resultado.

\section{TEST DE PRESIÓN DETRUSOR-FLUJ O MICCIONAL. \\ Urodinámica en el varón adulto con síntomas del tracto urinario inferior}

Uno de los retos prácticos más importantes que tiene ante si la urodinámica, es diferenciar aquellos pacientes que sufren sintomatología irritativaobstructiva, con obstrucción del tracto urinario inferior, comúnmente causada por adenoma de próstata, de aquellos otros que padeciendo los mismos síntomas, no presentan obstrucción.

La H.B.P. es una enfermedad con una prevalencia clínica alta ${ }^{40-42}$, constituyendo los gastos que se derivan de su tratamiento un problema económico social considerable, que se agravará en el futuro con el progresivo envejecimiento de la población ${ }^{43,44}$. 
Los sintomas que la H.B.P. ocasiona no son patognomónicos, sino que pueden también deberse a cualquier otro proceso obstructivo urinario inferior o incluso a un proceso de envejecimiento ${ }^{45,46}$. Un porcentaje no despreciable operados por H.B.P., no muestran obstrucción urinaria en el estudio urodinámico realizado ${ }^{47}$.

La realización de pruebas diagnósticas objetivas, que confirmen el diagnóstico de H.B.P. y que sirvan como indicación del tratamiento a seguir adquiere en este contexto una especial relevancia.

La importancia de determinar si existe o no obstrucción radica en la posibilidad de evitar la cirugía a los pacientes no obstruidos, en los que la sintomatología podría deberse a otras causas: afectación vesical por la edad, inestabilidad vesical y urgencia sensorial fundamentalmente.

El estudio urodinámico, determinación simultánea de presión vesical y flujo, permite clasificar los pacientes en tres grupos:

a) Obstruidos (presión elevada y flujo bajo).

b) No obstruidos (presión normal y flujo normal).

c) Contractilidad afectada (débil contracción y flujo bajo).

El coste económico que supone el estudio urodinámico y lo invasivo del mismo ha hecho que se intente discriminar estas situaciones mediante la flujometría simple. El flujo máximo se correlaciona mejor que los sintomas con la obstrucción determinada con exploración urodinámi$\mathrm{ca}^{48}$. Permite distinguir entre los pacientes del grupo b, (varones normales), y los del grupo a y c (obstruidos y afectación contráctil), pero no es capaz de diferenciar entre estos dos últimos grupos. Estableciendo el nivel de corte en 12 $\mathrm{ml} . / \mathrm{seg} .{ }^{48,49}$. Sólo el 3\% de los pacientes diagnosticados de H.B.P. e intervenidos no estarian obstruidos $^{50}$.

De acuerdo con esto, el flujo máximo parece tener valor pronóstico en cuanto a los resultados quirúrgicos se refiere. Aquellos pacientes con un flujo inferior a $15 \mathrm{ml} . / \mathrm{seg}$. experimentan una mejoría clínica superior a la de aquellos otros con un flujo máximo superior a dicho valor ${ }^{51}$.

La determinación de presiones simultáneamente con la flujometría distingue claramente entre pacientes obstruidos (presión alta y flujo bajo) de aquellos con afectación detrusoriana (pre- sión baja y flujo bajo), permitiendo así descartar la cirugía en todo este grupo, incluidos los pacientes que la flujometría aislada no es capaz discriminar $^{52}$. Para definir mejor la obstrucción prostática han sido aplicados sofisticados modelos matemáticos. Su superioridad, sin embargo, a la hora de definir la indicación quirúrgica y predecir el éxito de la misma no está clara ${ }^{53,54}$.

El estudio urodinámico es incapaz de separar entre la descompensación detrusoriana consecuencia de la edad y aquella otra originada por una situación obstructiva prostática crónica. Ambas se traducen urodinámicamente por presiones vesicales y flujos máximos disminuidos. En este sentido, es imposible predecir individualmente que paciente concreto mejorará tras la intervención. Es cierto que el porcentaje de respuesta es menor en el grupo de afectación detrusoriana (presiones y flujos bajos) que en el obstruido (presiones altas y flujos bajos), pero no es menos cierto que un número no despreciable de los primeros experimentan también la desaparición de sus síntomas ${ }^{51,55-58}$.

Una situación especial la representa aquella en la que existe sintomatología clínica con flujos superiores a $15 \mathrm{ml} / \mathrm{seg}$. Ésta puede estar causada por obstrucción a flujo alto (flujo alto y presiones elevadas) o por otras causas, entre ellas inestabilidad vesical. La no demostración urodinámica de presiones altas y por tanto de obstrucción inferior condiciona el éxito quirúrgico y hay que ser muy prudente a la hora de hacer la indicación operatoria en estas condiciones. El estudio urodinámico encuentra aquí una de sus indicaciones más claras ${ }^{59}$.

La inestabilidad vesical como tal no es causa de exclusión quirúrgica. Existe preoperatoriamente aproximadamente en el 60\% de los pacientes, siendo la incidencia post-operatoria del 20$30 \%$. No es posible predecir la desaparición o la persistencia de la misma tras la cirugia ${ }^{60}$.

El estudio urodinámico es obligado en los pacientes con alguna afectación que pueda ocasionar neuropatía vesical (A.C.V.A., Parkinson, diabetes, amputación abdómino-perineal, etc.). El conocimiento de la situación miccional (hiperactividad, hipoactividad, disinergia, existencia o no de obstrucción) nos permitirá tomar la decisión correcta $^{61}$. 
El estudio urodinámico lo realizamos también en aquellos pacientes jóvenes con un diagnóstico de presunción de disectasia cervical. Lo aseguramos así antes de proceder al tratamiento quirúrgico $^{62}$.

\section{Obstrucción femenina}

El diagnóstico de la obstrucción urinaria inferior femenina es problemático y no hay criterios urodinámicos universalmente aceptados de la misma. Massey y Abrams ${ }^{63}$ sugirieron que una presión miccional superior a $50 \mathrm{~cm} \mathrm{H}_{2} \mathrm{O}$, junto con un flujo inferior a $12 \mathrm{ml} / \mathrm{seg}$ o residuo significativo, podía indicar obstrucción. Esto posiblemente sea cierto, pero hay un gran porcentaje de mujeres diagnosticadas de obstrucción tras cirugía correctora de incontinencia que no cumplen estos criterios y, sin embargo, tras la uretrolisis mejoran. En nuestra propia experiencia, ninguna de nuestras pacientes en esta situación ha mostrado en el estudio urodinámico, tras la cirugía antiincontinencia, presiones superiores a $50 \mathrm{~cm} \mathrm{H}_{2} \mathrm{O}$ $\mathrm{y}$, sin embargo, han mejorado ostensiblemente tras la cirugía desobstructiva. Las presiones halladas han sido superiores, de una forma estadísticamente significativa, respecto a las previas a la cirugía anti-incontinencia. Sin embargo, los valores encontrados son relativamente bajos y por sí solos parece problemático establecer un nivel donde la presión indique obstrucción. Las presiones vuelven a descender de forma estadísticamente significativa tras la uretrolisis, lo que junto con el dato anterior, parece sugerir que existe una respuesta detrusoriana femenina a la obstrucción y a la posterior desobstrucción, aunque los valores sean inferiores a los encontrados en el varón. La significación estadística no significa relevancia clínica en esta situación.

La flujometría aislada puede jugar un papel muy importante en el diagnóstico de la obstrucción tras cirugía de la incontinencia femenina. Awad $^{64}$ encuentra en los pacientes con inestabilidad y sintomatología tras sling una disminución significativa del flujo urinario. Farrar ${ }^{65}$ piensa que un flujo inferior a $15 \mathrm{ml} / \mathrm{seg}$ con presiones normales o bajas puede ser indicativo de obstrucción urinaria. Kuo ${ }^{66}$ no encuentra variación en los flujos máximos en los pacientes que tras sling no presentan sintomatología. Al igual que sucede con las presiones, el flujo aislado no permite diagnosticar la obstrucción, pero comparando con el flujo previo a la cirugía permite apoyar dicho diagnóstico. Todas nuestras pacientes obstruidas han mostrado un flujo máximo post-cirugía claramente inferior al realizado antes de la intervención.

La combinación de presión detrusoriana a flujo máximo, flujo máximo y presencia de residuo urinario puede dar lugar a un nomograma para diagnosticar la obstrucción urinaria femenina. Sin embargo, para aceptarlo como cierto debe cumplir la condición de vuelta a la normalidad de los valores tras la acción terapéutica. Unos puntos de corte suficientemente restrictivos clarifican casi con exactitud la obstrucción anatómica, pero dejan sin diagnosticar casos. De cualquier manera, no es lo mismo manejar una paciente conociendo la existencia de una posible causa obstructiva previa, como es la cirugía anti-incontinencia, que intentar resolver un caso sin causa obstructiva anterior conocida.

\section{VIDEOURODINÁMICA}

La videourodinámica combina simultáneamente la evaluación del conjunto vejiga-cuello vesical-uretra. Define la posición, morfología y funcionalidad de estos componentes, siendo la técnica más sofisticada, costosa y exacta, constituyendo el patrón oro para el diagnóstico en algunos casos. Requiere el registro simultáneo de la presión vesical, abdominal y del detrusor. Precisa de infusión de medio de contraste y fluoroscopia, lo que permite ver el efecto de las maniobras de esfuerzo y su repercusión. A su vez se puede evaluar el nivel de la continencia urinaria-fuga (proximal, medio-uretral o ausente), así como los cambios anatómicos (cistocele, cuello vesical, hipermovilidad, déficit esfinteriano, disfunción neurógena etc...). No todos los centros se pueden permitir realizar estudios de este tipo, por disponibilidad de tecnología, personal y medios que nos lleven al diagnóstico. Reviste particular utilidad en el estudio de la disfunción neurógena vesicouretral, dado que establece el diagnóstico más exacto y fisiopatológico, a efectos del tratamiento oportuno ${ }^{67}$. No está claro que su uso mejore el resultado del tratamiento, ni que sea superior su capacidad diagnóstica al estudio urodinámico más CUMS realizados de forma aislada. 


\section{DISFUNCIÓN NEURÓGENA VESICOURETRAL}

La urodinámica sigue siendo uno de los pilares para el diagnóstico y control evolutivo de tratamiento de este importante y diverso grupo de enfermos. Permite un diagnóstico funcional exacto, que posibilita un tratamiento correcto, encaminado a conseguir los dos fines importantes del mismo: la preservación renal y la continencia urinaria.

\section{NE UROMODULACIÓN}

Las interacciones y relación entre el sistema nervioso autónomo y somático general es una parte integral del control correcto del mecanismo de vaciamiento y de la estabilidad de la vejiga. Alguna de estas interacciones reflejas son inhibidores mientras que otras son facilitadoras. Se requiere un perfecto balance entre ellos para un perfecto vaciado y mantener una vejiga estable. Los síndromes con disfunción del vaciado se asocian con cierto grado de labilidad del comportamiento del reflejo sacro. Por lo tanto, cuando se inducen o se emplea la interacción de los reflejos se modula el suelo de la pelvis y la inestabilidad del detrusor. En 1965, Habib ${ }^{68}$ expone los resultados de la estimulación eléctrica de raíces sacras S3 y S4, instalando electrodos de platino unilateralmente vía transperitoneal en dichas raíces. Estos electrodos los conecta a un estimulador subcutáneo, pero fracasa en el intento porque la fibrosis perineural que aparece en el electrodo, hace que deje de ser eficaz dicha estimulación. Tanago ${ }^{69}$ desarrolla posteriormente el modelo clínico superando estos problemas. En la actualidad, la neuromodulación de raíces sacras en la última década se ha ubicado, con unas estrictas indicaciones, como una modalidad de tratamiento que da respuesta a enfermos con síndrome urgenciafrecuencia o a trastornos del vaciado, refractario a otras modalidades de tratamiento.

La retención urinaria crónica idiopática no obstructiva, es una entidad que existe, aún cuando así definida sea poco frecuente, a la cual sólo se le podía ofrecer como tratamiento el cateterismo vesical intermitente. La neuromodulación de raíces sacras representa en estos casos, en los que hay indemnidad del arco reflejo sacro, una modalidad de tratamiento efectiva, mejorando su calidad de vida y haciendo desaparecer las infeccio- nes del tracto urinario. Otros síndromes de urgencia frecuencia, incontinencia de urgencia y de dolor pélvico crónico se han podido ver mejorados con esta modalidad terapéutica.

Dentro del Grupo Nacional de Urodinámica, la Neuromodulación de raíces sacras ha sido puesta en marcha ${ }^{70}$. Queda todavía por ver sus resultados a largo plazo y el tiempo establecerá sus indicaciones concretas.

\section{URODINÁMICA AMBULATORIA}

Las indicaciones generales, según el informe de estandarización ${ }^{71}$, se establecen para aquellos sintomas del tracto urinario inferior que una investigación convencional urodinámica no permite reproducir o explicar, en aquellas situaciones en las que la urodinámica convencional no pueda ser satisfactoria, en algunos casos de disfunción neurogénica del tracto urinario inferior y en la evaluación de terapéuticas para la disfunción del tracto urinario inferior.

Supone un complemento a la urodinámica convencional muy útil, fundamentalmente para aquellos casos en los que el diagnóstico urodinámico es no reproducción de síntomas, incontinencia urinaria multifactorial compleja, antes de enterocistoplastia en vejiga de baja acomodación, previa a implante definitivo de neuromodulador de raíces sacras y para aquellos enfermos, en los que el estudio convencional proporciona resultados no reproducibles o artefactuales. Está demostrado que en muchos casos de disminución de acomodación, ésta refleja contracciones fásicas de larga duración, sin corresponder necesariamente a una acomodación disminuida. Tiene como ventaja ser más fisiológica, menos molesta, es un equipo de urodinámica en toda regla, estudia varios ciclos miccionales. Como inconveniente destacariamos el costo y su disponibilidad.

$\mathrm{El}$ informe debe ajustarse a las indicaciones urodinámicas y debe incluir:

- Duración del registro.

- Señal/descripción de la calidad de los datos.

- Ritmo de llenado, tiempo, método, y volumen de cualquier llenado retrógrado antes de comenzar la monitorización.

- Dosis y pauta de diuréticos, si son administrados.

- Volumen de líquido ingerido durante el test. 
- Número de vaciados.

- Volumen total y rango de los vaciados y del residuo urinario post-miccional.

- Episodios de urgencia, incontinencia urinaria y dolor.

- Actividad del detrusor durante la fase de llenado (frecuencia, horario, duración, amplitud, área, forma).

- Análisis de la presión/flujo.

- Resultados de las maniobras de provocación empleadas durante el test.

- Razones para la finalización del registro si se finaliza prematuramente.

El protocolo de la monitorización debe incluir:

- Duración planeada del registro.

- Duración real del registro.

- Señal/valoración de la calidad de los datos.

- Especificaciones del aparato de registro, es decir, fabricante, tipo, niveles de muestreo, botones de sucesos.

- Catéter: tipo, transductor, localización, vía, y técnica de fijación.

- Aparato o método de detección de pérdidas urinarias.

- Transductor de flujo urinario.

- Protocolo de diuresis.

- Volumen y horario de ingesta de líquidos durante el test.

- Dosis y pauta de administración de diuréticos.

- Ritmo de llenado, tiempo, método y volumen de cualquier llenado retrógrado antes de comenzar o durante la monitorización.

- Sucesos registrados por diarios, o marcadores electrónicos.

- Actividad del detrusor durante la fase de llenado asociada a cualquier urgencia, incontinencia y dolor.

- Análisis de presión/flujo (de acuerdo a las normas de la ICS).

- Cualquier maniobra de provocación empleada durante el test.

- Razones para la finalización del registro si se finaliza prematuramente.

- Las presentaciones de las curvas urodinámicas deberían incluir la identificación del canal, las unidades de medida, la escala mínima para las presiones debería ser $2 \mathrm{ml} / 5 \mathrm{~cm}$ $\mathrm{H}_{2} \mathrm{O}$ y la escala mínima para el tiempo debería ser de $4 \mathrm{~cm} /$ minuto.
En nuestra experiencia la monitorización ambulatoria supone un complemento a la urodinámica convencional para minimizar la no reproducción de síntomas ${ }^{5,72,73}$, permite estudiar con mayor fiabilidad el vaciado en la mujer y sobre todo puede ser muy útil en la determinación de la acomodación en la disfunción neurógena vesicouretral. En las Figuras 9, 10 y 11 se muestra un monitor en enferma, como se transfieren los datos $\mathrm{y}$ un ejemplo de registro.

\section{CONTROVERSIAS Y RETOS DE FUTURO}

Entre otros problemas que quedan dentro del campo de la urodinámica sin resolver son:

1. El exacto conocimiento de la alteración de la transmisión de presiones en la incontinencia de esfuerzo femenina ayudará a su mejor manejo.

2. La aclaración de las alteraciones últimas en las disfunciones miccionales en la infancia y en el Sindrome de Hinman sigue siendo un punto importante de estudio.

3. Continuamos sin saber la causa exacta de la inestabilidad vesical.

4. La determinación de parámetros fiables urodinámicos que de una forma precisa determinen o caractericen la obstrucción del tracto urinario en la mujer, sigue siendo un reto actual de la urodinámica. Tampoco están debidamente caracterizados por la misma razón, los trastornos de vaciado en la mujer en lo que se refiere a disfunción obstructiva de vaciado y déficit contráctil. Siempre estas condiciones han de sustentarse en parámetros además de urodinámicos en condiciones clínicas o signos de demostración objetiva.

5. La urodinámica ambulatoria, supone actualmente un importante complemento a la urodinámica convencional puesto que además de ser un equipo semejante al convencional, minimiza la no reproducción de síntomas. No obstante, el futuro de su utilidad se centra fundamentalmente en tres vertientes:

- En la determinación más precisa de la acomodación vesical en la disfunción neurógena vesicouretral.

- La evaluación urodinámica previa a la implantación de un neuromodulador.

- El estudio de las disfunciones de vaciado en la mujer, cuya fisiopatología y urodinámica no resuelve su actual reto clínico. 


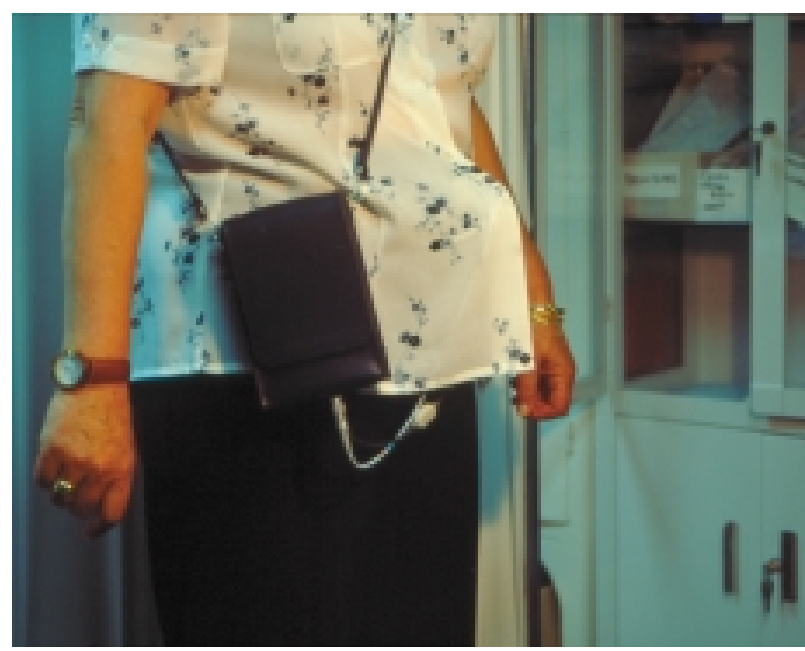

FIGURA 9. Monitor portable de urodinámica ambulatoria.

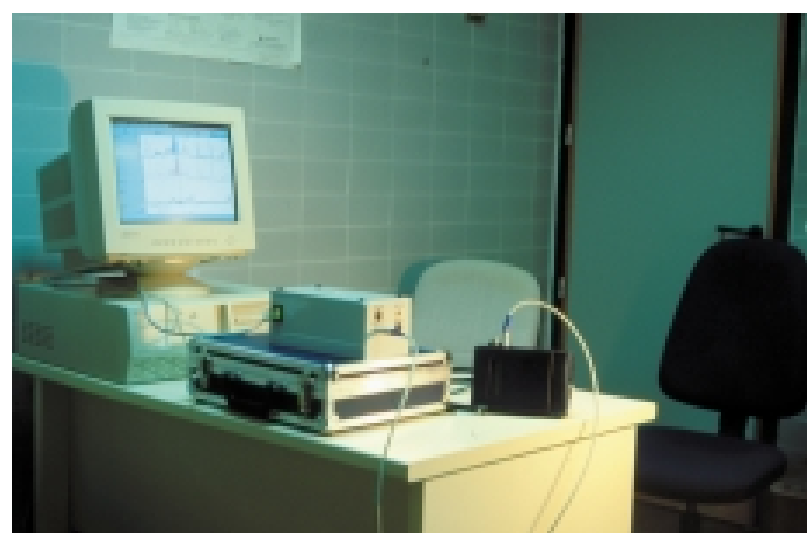

FIGURA 10. Los datos obtenidos del portable se transfieren $a$ un ordenador convencional.

6. Existe una gran controversia respecto a la indicación de la realización del estudio urodinámico en la mujer con incontinencia urinaria de esfuerzo, ya que la sintomatología demuestra una sensibilidad del $91 \%$, especificidad del $51 \%$ y un valor predictivo positivo del $75 \%{ }^{74}$. El estudio urodinámico permite reproducir el síntoma, descartar inestabilidad vesical, evaluar disfunciones obstructivas de vaciado asociadas a prolapso, permite conocer la presión abdominal de fuga, teniendo una morbilidad discreta en estos casos y un coste establecido.

La controversia actual se orienta a encontrar las indicaciones precisas para la indicación del estudio urodinámico:

- La Agencia de Política Sanitaria e Investigación (Agency of Healt Care Policy and Research) desarrolló guías clínicas para el tratamiento de la incon-

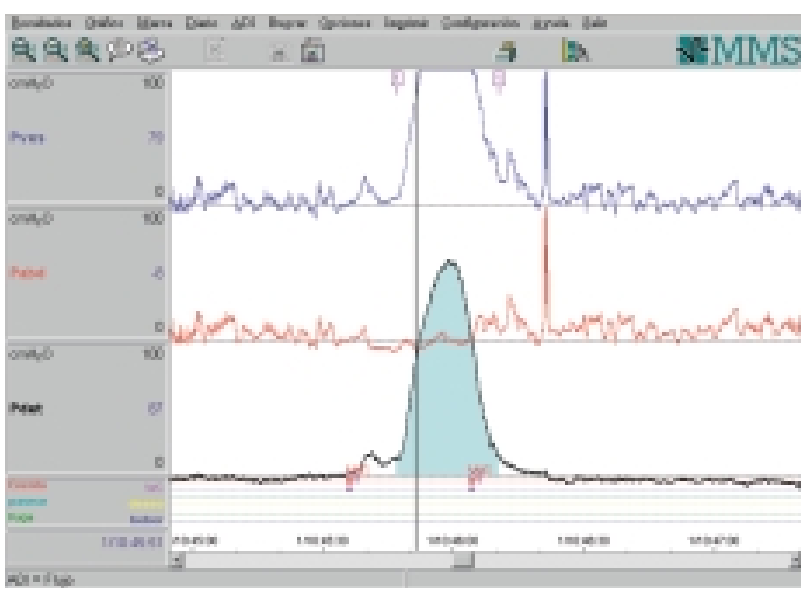

FIGURA 11. Inestabilidad vesical detectada con un monitor ambulatorio que no se reprodujo en un estudio convencional.

tinencia urinaria y en la incontinencia urinaria de esfuerzo no complicada en mujeres, no se recomendaba de forma rutinaria la realización de estudios urodinámicos. Sólo se reservaban para factores agravantes y comorbilidades. Existen estudios randomizados en los que se demuestran que no se obtiene beneficio por la realización del estudio urodinámico en mujeres en las que se instauran tratamientos conservadores para la incontinencia urinaria de esfuerzo ${ }^{75}$.

- Trabajos retrospectivos que establecen un análisis de decisión (posiblemente con cierto sesgo) reflejan que el estudio urodinámico previo al tratamiento quirúrgico por vía retropúbica no influye en el resultado ${ }^{75}$.

- La revisión de la Fundación Cochrane, aplicando la Medicina Basada en la Evidencia, intentó aclarar el beneficio obtenido por la realización del estudio urodinámico en el tratamiento de la incontinencia urinaria de esfuerzo y concluye que no supone beneficio para el tratamiento de la incontinencia urinaria de esfuerzo en pacientes en las que se indicó tratamiento conservador. No se pronuncia respecto al tratamiento quirúrgico, al no existir ensayos aleatorizados, que no se basen en modelos simulados, y que comparen el diferente efecto de diferentes estrategias de estudios previos a la cirugía sobre los resultados clínicos a obtener.

- La $2^{\text {nd }}$ International Consultation on Incontinence ${ }^{74}$ avalada por la OMS, en relación a la capacidad del estudio urodinámico para mejorar el resultado de los tratamientos para la incontinencia, reconoce que dados los estudios exis- 
tentes el nivel de evidencia científica es de $4 / 5$ (series de casos y opinión de expertos), recomendando el tratamiento conservador en base a diagnóstico clínico. Recomienda el estudio urodinámico si falla el tratamiento conservador, sospecha de disfunción neurógena, disfunción de vaciado, o previamente a tratamiento quirúrgico.

7. La aclaración de las indicaciones de la neuromodulación, circunstancia que actualmente se está desarrollando, hará que se perfile de forma más absoluta su indicación y que su accesibilidad esté por encima de la geografía en la que se encuentra el enfermo.

8. Sistemas de inteligencia artificial, son herramientas que pueden tener cara al diagnóstico urodinámico; utilidad en cuanto al perfeccionamiento del diagnóstico, miniaturización, mayor precisión de los equipos y sistemas más fisiológicos son los objetivos que persigue la investigación en este campo.

9. El esfinter artificial: la obtención de materiales biocompatibles que mejoren su vida media sigue siendo actualmente un objetivo para mejorar su tolerancia.

10. El desarrollo de tejidos que permitan realizar ampliaciones vesicales, sin los problemas inherentes al intestino, es otro reto de futuro que además estimula la carente investigación básica y experimental en urodinámica.

11. En el capítulo de prevención, aunque de su incidencia existen datos objetivos de disminución, siguen apareciendo casos de mielodisplasia. Tanto la investigación, como medidas de aplicación reales de toma de ácido fólico y realización de ecografías de alta resolución siguen siendo necesarias.

\section{REFERENCIAS}

1. CONEJERO J.: Urodinámica Aplicada. Ed por J. Conejero. Ed MCR, Barcelona, 1987. Prólogo.

2. SALINAS J.: Memoria Docente, 1990. Comunicación personal.

3. LLORÉNS FJ, COLLADO A.: Flujometría: técnica, aplicaciones y datos. En Urodinámica Clínica $3^{\text {a }}$ Ed. J. Salinas, J. Romero. Madrid. Ed. Luzán 2002; 5: 137.

4. ABRAMS PH.: Urodynamic techniques. En: Abrams, P. Urodynamics. 2nd edition. Springer 1997: 20-39.

5. PUERTAS M, PRIETO L, ROMERO J.: Urodinámica ambulatoria: concepto y técnicas. En Urodinámica Clínica $3^{\mathrm{a}}$ Ed. J. Salinas, J. Romero. Madrid. Ed. Luzán 2002; 13: 435.
6. SALINAS J, VIRSEDA M, ARREDONDO F y cols.: Estudio de la validez de la flujometría con manguito como técnica diagnóstica de obstrucción del tracto urinario inferior en el varón. Com. 136. Cogreso Nacional de Urología. Granada, 2001; 13-16/5.

7. KINN AC.: Stop-flow measurement of detrusor contractility in bladder outflow obstruction. $\mathrm{Br} J$ Urol 1989; 64: 363-367.

8. PORENA M, CONSTANTINI E, ROCIOLA W, MEARINI E.: Biofeedback successfully cures detrusorsphincter disinergia in pediatric patients. $J$ Urol 2000 jun; 163 (6): 1927-1931.

9. STEPHENSON TP.: The interpretation of convencional urodynamics. En: Mundy AR, Stephenson TP, Wein AJ. Urodynamics. Principles, practice and application. Churchill Livingstone 1994: 111-132.

10. JENSEN KM, BRUSKEWITZ RC, IVERSEN P, MADSEN PO.: Abdominal straining in benign prostatic hyperplasia. J Urol 1983 jan; 129 (1): 44-47.

11. TOMITA Y, OGAWA A.: Uroflowetry combined with simultaneous measurement of abdominal pressure by tocodynamometer. J Urol 1993 feb; 149 (2): 335338.

12. MEFFAN PJ, NACEY JN, DELAHUNT B.: Effect of abdominal straining on urinary flow rate in normal males. Br J Urol 1991 feb; 67 (2): 134-139.

13. RAPARIZ M, SALINAS J.: Síndrome de micción no coordinada. Nuevos aspectos de un viejo problema. Actas Urol Esp 1995 abr; 19 (4): 261-280.

14. BARRETT DM, WEIN AJ.: Flow evaluation and simultaneous external sphincter electromyography in clinical urodinaminamics. J Urol 1981 apr; 125 (4): 538-541.

15. CHAUDHURI TK, FINK S, BURGER RH et al.: Physiological considerations in radionuclide urodynamic studies. Am J Physiol Imaging 1989; 4 (2): 70-74.

16. GROSHAR D, EMBON OM, SAZBON A et al.: Radionuclide assessment of bladder outlet obstruction: a noninvasive (1-step) method for measurement of voiding time, urinary flow rates and residual urine. J Urol 1988 feb; 139 (2): 266-269.

17. GROSHAR D, KORITNY ES, EMBON OM et al.: Radionuclide measurement of bladder emptying rate. Simultaneous comparison with urinary flow rate obtained by uroflowmetry. Clin Nucl Med 1988 may; 13 (5): 363-365.

18. LIDGI S, MOSKOVITZ B, GROSHAR D et al.: Preoperative prediction of results of prostatectomy by a symptom score and radionuclide uroflowmetry. Eur Urol 1989; 16 (2): 97-100.

19. MAYR B, SWOBODA G, LEISNER B et al.: Comparative studies of radioisotope uroflowmetry (author's transl). Urologe A 1979 may; 18 (3): 133136.

20. CORLETT RC, ROY S.: Carbon dioxide uroflowmetry. J Urol 1979 oct; 122 (4): 512-514.

21. EDWARS LE, POWELL CS.: Urodynamic studies: an unsual complication. Br J Urol 1986; 58: 461-462.

22. ICS: Standarisation of terminology oflower urinary tract function. Neurourol and Urodyn 1988; 7: 403426.

23. BLAIVAS JG.: Obstrucción del tracto urinario inferior en el varón. Clin Urol North Am (Ed. Española) 1996; 23 (3): 387. 
24. ABRAMS P, BLAIVAS J, NORDLING $J$ et al.: The objective evaluation of bladder outflow obstruction. In In the 2nd International consultation on Benign Prostatic Hyperplasia (BPH). Edited by A.T.K. Cockett, Y. Aso, C. Chatelain. Paris. Wordl Healt Organization 1993; 6: 153.

25. McGUIRE EJ, CESPEDES RD, O'CONNELL HE.: Leak-point pressures. Urol Clin North Am 1996; 23: 253-262.

26. McGUIRE EJ.: Clinical assessment of urethral sphincter function. J Urol 1993; 150: 1452-1454.

27. PRIETO L, LLORÉNS FJ, GARCÍA LÓPEZ F.: Urodinámica en la incontinencia urinaria femenina tipo III. Urol Integr Invest 2000; 5 (3): 233-244.

28. FLOOD HD, ALEVIZATOS C, LIU JL.: Sex differences in the determination of abdominal leak point pressure in patients with intrinsic sphincter deficiency. J Urol 1996; 156: 1737-1740.

29. MIKLOS JR, SZE EH, KARRAM MM.: A critical appraisal of the methods of measuring leak-point pressures in women with stress incontinence. Obstet Gynecol 1995; 86: 349-352.

30. DUPONT MC, ALBO ME, RAZ S.: Diagnosis of stress urinary incontinence. An overwiew. Urol Clin North Am 1996; 23 (3): 407.

31. NITTI VW, COMBS AJ.: Correlation of Valsalva leak point pressure with subjective degree of stress urinary incontinence in women. J Urol 1996; 155: 281.

32. RAZ S, ERIKSON DB.: SEAPI-QMN incontinence classification system. Neurourol and Urodynam 1992; 11: 187.

33. M. LEVA VALLEJO M, ANGLADA F, REGUEIRO LÓPEZ JC y cols.: Presión abdominal de fuga en la evaluación de la incontinencia urinaria de esfuerzo femenina: relación con parámetros clínicos y urodinámicos. Urodinámica Aplicada 2000; 13-2: 124.

34. HORBACH NS, OSTERGARD DR.: Predicting intrinsic urethral sphincter dysfunction in women with stress urinary incontinence. Obstet Gynecol 1994; 84 (2): 188-192.

35. SULTANA CJ.: Urethral closure pressure and leakpoint pressure in incontinent women. Obstet Gynecol 1995; 86: 839-842.

36. SWIFT SE, OSTERGARD DR.: A comparison of stress leak-point pressure and maximal urethral closure pressure in patients with genuine stress incontinence. Obstet Gynecol 1995; 85: 704-708.

37. PALAO YAGO F.: Validación del diagnóstico clínico y de los perfiles de presión uretral en el diagnóstico de la incontinencia urinaria femenina de esfuerzo. Tesis Doctoral. Facultad de Medicina. Universidad de Granada, 1997.

38. PALAO YAGO F, GALISTEO R, JIMÉNEZ A y cols.: Relación entre la presión uretral de cierre y la presión abdominal de fuga en la incontinencia urinaria femenina de esfuerzo. Urodinámica Aplicada 2000; 13 (1): 20-28.

39. CAMMU H, VAN NYLEN M, AMY JJ.: Diez años de seguimiento de los ejercicios de Kegel de la musculatura del suelo pélvico para el tratamiento de la incontinencia de esfuerzo genuina. B.J.U. International 2000; 85: 655-688.

40. BERY SJ, COFFEY DS, EWING LL.: The development of human benign prostatic hypaerplasia with age. J Urol 1984; 132: 474-479.
41. FERNÁNDEZ E, PORTA M, ALONSO J et al.: Epidemiology of prostatic disorders in the Ciyi of Barcelona. Int $J$ Epidemiol 1992; 21: 959-965.

42. CHICHARRO JA, BURGOS R, SÁNCHEZ JJ y cols.: Prevalence of benign prostatic hyperplasia in spanish men 40 years old or older. J Urol 1998; 159: 878-882.

43. HOLTGREWE HL.: Economics of benign prostatic hyperplasia. En Kirby R. Textbook of benign prostatic hyperplasia 1996. Isis Medical Media Ltd Oxford; 44: 527-536.

44. UNDA URZÁIZ M, RODRÍGUEZ ALCÁNTARA F, BADÍA X y cols.: Características sociosanitarias $\mathrm{y}$ diagnósticas del paciente prostático en España a finales del siglo XX. Actas Urol Esp 2001; 25: 200206.

45. MADSEN FA, BRUSKEWITZ RG.: Clinical manifestations of benign prostatic hyperplasia. Urol Clin North Am 1995; 22: 291-298.

46. HOLM NR, HORN T, HALD T.: Detrusor in ageing and obstruction. Scand J Urol Nephtol 1995; 29: 459.

47. SCHAFER W, RUBBEN H, NOPPENEY R et al.: Obstructed and unobstructed prostatic obstruction. A plea for urodynamic objetivation of bladder outflow obstruction in benign prostatic hyperplasia. World J Urol 1989; 6: 198-203.

48. SHOUKRY I, SUSSET JG, ELÑHIALAI MM et al.: Role of uroflowmetry in the assesment of lower urinary tract obstruction in adult males. Br J Urol 1975; 47: 559-566.

49. CHANCELLOR MB, BLAIVAS JG, KAPLAN SA et al.: Bladder outlet obstruction versus impaired detrusor contractility: the role of uroflow. J Urol 1991; 145: 810-812.

50. McLOUGHLIN J, GILL KR, ABEL PD et al.: Symptoms versus flow rates versus urodynamics in the selection of patients for prostatectomy. Br J Urol 1990; 66: 303-305.

51. JENSEN KM, JORGENSEN JB, MOGENSEN P.: Urodynamics in prostatism. Prognostic value of uroflowmetry. Scan J Urol Nephrol 1988ª; 22: 109-117.

52. SALINAS J, VIRSEDA M.: Los estudios urodinámicos en la valoración de la hiperplasia benigna prostática. En Salinas J. Romero (edit) Urodinamica Clínica. Madrid. Jarpio Editores 1995; 13: 205-208.

53. SCHAFER W, RUBBEN H, NOPPENEY R et al.: Obstructed and unobstructed prostatic obstruction. A plea for urodynamic objetivation of bladder outflow obstruction in benign prostatic hyperplasia. World J Urol 1989; 6: 198-203.

54. ROLLEMA HJ, VAN MASTRIGT R.: Objetive analysis of prostatism: a clinical application af the computer program CLIM. Neurourol Urodyn 1991; 10: 71-76.

55. DONOVAN JL. ABRAMS P, SCHAFER W.: The International Continence Society study on BPH: urodynamic quality control and data analysis. J Urol 1994; 151: 294 .

56. KIRCHNER-HERMANS R, THORNER M, SAHAFER W et al.: Reproductibility of urodynamic data in BPH: influence of patients and investigator on data quality and analysis. $J$ Urol 1994; 151: 295 ${ }^{\text {a }}$.

57. NEAL DE, RAMSDEN PO, SHARPLES L et al.: Outcome of elective prostatectomy. Br Med J 1989; 299: $762-767$. 
58. KUO HC, TSAI YTC.: The predictive value of urine flow rate and voiding pressure in the operative outcome of benign prostatic hypertrophy. Tiwan I Hsueh Hui Tsa Chih 1988; 87: 323-330.

59. JENSEN KM, ANDERSEN TJ.: Urodynamic implications of benign prostatic hyperplasia. Urologoe 1990; 29: 1-4.

60. OLSSON CA, GOLUBOFF ET, CHANG D et al.: Urodynamics and the etiology of post-prostatectomy urinary incontinence. J Urol 1994; 155: 326 ${ }^{\text {a }}$.

61. PERALES L, FERNÁNDEZ E, JIMÉNEZ M y cols.: Evaluación urodinámica y tratamiento de los pacientes con adenoma de próstata asociado a neuropatía vesical. Urol Integ Invest 1998; 3: 38-41.

62. ROMERO MAROTO J, TALLADA BUÑUEL M, VERDÚ TARTAJO F y cols.: Functional evaluation of bladder neck disease. Eur Urol 1982; 8: 269-273.

63. MASSEY JA, ABRAMS PH.: Obstructed voiding in the female. Br J Urol 1988; 61: 36.

64. AWWAD SA, FLOOD HD, ACKER KL.: The significance of prior anti-incontinence surgery in women who present with urinary incontinence. $J$ Urol; 140: 514-517.

65. FARRAR DJ, OSBORNE JL, STEPHENSON TP, WHITESIDE CG, WEIR J, BERRY J, MILROY EJG, TURNER WARWICK RA.: Urodynamic view of bladder outflow obstruction in the female: factors influencing the results of treatment. $B r J$ Urol 1976; 47: 815.

66. KUO H.: Comparison of video urodynamic results after the pubovaginal sling procedure using rectus fascia and polypropylene mesh for stress urinary incontinence. J Urol 2001; 165: 163-168.

67. SALINAS J, PRIETO L, PÁEZ A, DIEGO A, RAPARIZ M, SILMI A, RESEL L.: Nueva clasificación del reflujo vesicoureteral. Archv Esp Urol 1992; 45 (5): 449 453.

68. BORS E, COMARR EA.: Neurological methods ot treatment. In Neurological Urology, Physiology of micturition, Its Neurological disorders and sequelae. Ed. by Bors E. and Comarr EA. University Park Press, Baltimore 1971: 280.

69. TANAGO EA, SCHMIDT RA.: Electrical stimulation in the clinical management of the neurogenic bladder. J Urol 1988; 140: 1331.
70. RUIZ JL, ARLANDIS JF, JIMÉNEZ JF.: Neuromodulación mediante estimulación eléctrica de raíces sacras. En Neuromodulación, una nueva alternativa terapéutica para los trastornos del tracto urinario inferior. Ed. por Ruiz JL, Martínez E, Arlandis S, Jiménez JF. Tema Monográfico del Congreso Nacional de Urología. Ene Ediciones. Madrid 2000 mayo; 6: 147.

71. VAN WAALWIJK E, VAN DOORN, ANDERS K, KHULLAR V, KULSENG-HANSSEN S, PESCE F, ROBERTSON A, ROSARIO D, SCHÄFER W.: Estandarización de la monitorización urodinámica ambulatoria: informe del subcomité de estandarización de la Sociedad Internacional de Continencia para los estudios urodinámicos ambulatorios. Neurourology and Urodynamics 2000; 19: 113-125.

72. PUERTAS M, PRIETO L, ROMERO J.: No reproducción de síntomas en estudio urodinámico convencional. Urodinámica Aplicada 2000; 12 (3).

73. PUERTAS M, PRIETO L, ROMERO J.: Urodinámica ambulatoria, aplicaciones y datos. En urodinámica clínica. Tomo I. $3^{\text {a }}$ Edición ed. por J. Salinas, J. Romero. Madrid. Luzán Ediciones 2002; 13: 435.

74. HOMMA Y, BATISTA J, BAUER S, GRIFFITHS D, HILTON P, KRAMER G, LOSE P, POSIER P.: Urodynamics. In Incontinencia, $2^{\text {nd }}$. International Consultation Incontinence. Ed. By Abrams P, Cardozo L, Khoury S, Wein A. Paris, 2001: 319.

75. WEBER AM, TAYLOR RJ, WEI JT, LEMACK G, PIEDEMONTE MR, WALTERS MD.: Relación coste-eficacia de las pruebas preoperatorioas (evaluación básica en la consulta frente a urodinámica) en la incontinencia urinaria de esfuerzo en la mujer. B.J.U. International. Edición Española. Junio, 2002; 5 (3): 174

Dr. J. Romero Maroto

Hosp. Universitari Sant Joan D'Alacant

(Servicio de Urología)

Ctra. Nnal. 332 Alicante-Valencia s/n

03550 San Juan de Alicante (Alicante)

(Trabajo recibido el 21 noviembre de 2003) 